decreases in the occurrence of resistance to $46.7 \%$ and $28.1 \%$ among $E$ faecium and $E$ faecalis isolates from pigs, respectively. Erythromycin resistance among $E$ faecium isolates from broilers reached a maximum of $76.3 \%$ in 1997 , but decreased to $12.7 \%$ in 2000 concomitantly with more limited use of virginiamycin. Use of virginiamycin increased from 1995 to 1997 and was followed by an increased occurrence of virginiamycin resistance among $E$ faecium isolates in broilers-from $27.3 \%$ in 1995 to $66.2 \%$ in 1997. In January 1998, the use of virginiamycin was banned in Denmark, and the occurrence of virginiamycin resistance decreased to $33.9 \%$ in 2000 .

Use of avilamycin increased from 1995 to 1996 and was followed by an increase in avilamycin resistance among E faecium isolates from broilers, from $63.6 \%$ in 1995 to $77.4 \%$ in 1996 . Since 1996 , avilamycin usage has decreased, followed by a decrease in resistance to $4.8 \%$ in 2000 . These results show that it is possible to reduce the occurrence of antimicrobial resistance in a national population of food animals when the selective pressure is removed. Cases in which resistance to vancomycin was linked to resistance to erythromycin were exceptions. In such cases, resistance did not decrease until the use of both avoparcin and tylosin was limited.

FROM: Aarestrup FM, Seyfarth AM, Emborg HD, Pedersen K, Hendriksen RS, Bager F. Effect of abolishment of the use of antimicrobial agents for growth promotion on occurrence of antimicrobial resistance in fecal enterococci from food animals in Denmark. Antimicrob Agents Chemother 2001;45:2054-2059.

\section{AHRQ Issues Report on Evidence-Based Practice for Patient Safety}

The Agency for Healthcare Research and Quality (AHRQ) recently released a report, Making Health Care Safer, A Critical Analysis of Patient Safety Practices. The report reviews data on 79 specific patient safety practices. Among the 11 "highly proven" practices (mostly clinical interventions) are pre-operative administration of antibiotics to prevent infections and beta-blockers to prevent heart attacks during or after surgery. Researchers from the University of California at San Francisco, Stanford University, were commission by AHRQ to prepare the report for AHRQ. The report is based on a review of the medical literature on safety practices and on consultation with healthcare experts, focusing on issues relevant to care delivered in hospitals and on prevalent diseases and procedures. Practices were excluded if there were few or no scientific studies to support them, and more than a dozen practices long considered important by patient safety experts did not make the list.

The five chapters in the "Infection Control" section address handwashing compliance; barrier precautions; impact of antibiotic-use practices; and prevention of nosocomial urinary tract infections, intravascular catheterassociated infections, and ventilator-associated pneumonia. The chapters are all structured similarly, providing back- ground on the practice in question, opportunities for impact, study design and outcomes, evidence for effectiveness, potential for harm, cost of implementation, and a concluding comment. The narrative is followed by a tabular listing of studies, including description of the interventions, type of study design, and statistical results. Each chapter concludes with actual study citations.

AHRQ recognizes that there are gaps in each area, that more research is needed to determine which practices are most effective in improving patient safety, and that it needs to be determined how complex or costly they would be to implement. The National Forum for Health Care Quality Measurement and Reporting, a coalition of providers, consumers, purchasers, employers, and accrediting bodies, intends to use the report to develop a checklist of measures that patients may use to determine the actions hospitals and health facilities have taken to improve safety.

The report can be obtained by calling the AHRQ Publications Clearinghouse at (800) 358-9295 or sending an e-mail message to ahrqpubs@ahrq.gov. The report can be downloaded from the AHRQ web site as a pdf file, or viewed or printed online by chapter at www.ahcpr.gov/ clinic/ptsafety.

\section{Enterococcus faecalis Surface Protein and Urinary Tract Infections}

Enterococcus faecalis isolated from patients with bacteremia, endocarditis, and urinary tract infections more frequently express the surface protein Esp than do fecal isolates. Shankar and colleagues from the University of Oklahoma Health Sciences Center, Oklahoma City, conducted a study to assess the role of Esp in colonization and persistence of $E$ faecalis in an animal model of ascending urinary tract infection. They compared an Esp (+) strain of $E$ faecalis to its isogenic Esp-deficient mutant. Groups of $\mathrm{CBA} / \mathrm{J}$ mice were challenged transurethrally with $10^{8}$ colony-forming units of either the parent or mutant strain; bacteria in the urine, bladder, and kidneys were enumerated 5 days postinfection.

Significantly higher numbers of bacteria were recovered from the bladder and urine of mice challenged with the parent strain than from the bladder and urine of mice challenged with the mutant. Colonization of the kidney, however, was not significantly different between the parent and mutant strains. Histopathological evaluations of kidney and bladder tissue done at 5 days' postinfection did not show marked histopathological changes consistent with inflammation, mucosal hyperplasia, or apoptosis, and there was no observable difference between the mice challenged with the parent and those challenged with the mutant.

The authors concluded that, while Esp does not influence histopathological changes associated with acute urinary tract infections, it contributes to colonization and persistence of $E$ faecalis at this site.

FROM: Shankar N, Lockatell CV, Baghdayan AS, Drachenberg C, Gilmore MS, Johnson DE. Role of 
Enterococcus faecalis surface protein Esp in the pathogenesis of ascending urinary tract infection. Infect Immun 2001;69:4366-4372.

\section{Risk Factors for ICU Infection}

Appelgren and coinvestigators from the Karolinska Hospital, Stockholm, Sweden, conducted a study to identify risk factors for nosocomial infection in the intensive care setting and to provide a basis for allocation of resources. The study was a long-term prospective incidence study of risk factors for nosocomial infection in the surgical-medical intensive care unit of a university hospital.

A total of 2,671 patients were admitted during 4 years, and 562 of 574 patients staying $>48$ hours were observed during 4,921 patient-days (median length of stay 5 days; range, 2-114). Of these, 196 patients (34\%) had 364 nosocomial infections after the median 8 to 10 days, an infection rate of 14 of 100 admissions. Infection prolonged length of stay 8 to 9 days and doubled the risk of death. The infections were $17 \%$ bloodstream infections, $26 \%$ pneumonias, $34 \%$ wound infections, $10 \%$ urinary tract infections, and $13 \%$ other infections. The incidence of bloodstream infection declined significantly during the study years, from $12 \%$ to $5 \%$. In multiple regression analysis, the important variables for infection were central venous catheter, mechanical ventilation, pleural drainage, and trauma with open fractures.

High age, immunosuppression, and infection on admission did not influence the risk of acquiring infection. Trauma patients constituted $24 \%$ of the study population. Trauma with open fractures increased the risk of infection more than twofold $(P=.003)$, mainly due to wound infections.

The authors concluded that trauma patients with open fractures were the patients most at risk of infection, despite low disease severity scores. Resources to prevent nosocomial infection should be allocated to these patients.

FROM: Appelgren P, Hellstrom I, Weitzberg E, Soderlund V, Bindslev L, Ransjo U. Risk factors for nosoco- mial intensive care infection: a long-term prospective analysis. Acta Anaesthesiol Scand 2001;45:710-719.

\section{Staphylococcus epidermidis Biofilms}

The coagulase-negative staphylococci, in particular, Staphylococcus epidermidis, have emerged as major nosocomial pathogens associated with infections of implanted medical devices. These organisms, which are among the most prevalent bacteria of the human skin and mucous membrane microflora, present unique problems in the diagnosis and treatment of infections involving biofilm formation on implanted biomaterials. Epidemiological data that address whether invasive $S$ epidermidis strains can be traced to commensal organisms or an endemic occurrence of distinct strains with enhanced virulence have important implications for the implementation of appropriate infection control measures.

An extracellular polysaccharide adhesin represents a key virulence determinant in $S$ epidermidis and is required for biofilm formation. Production of this adhesin, which is encoded by the ica operon, is subject to phase variable regulation (ON $<>$ OFF switching). Recent advances in understanding the molecular events controlling polysaccharide adhesin synthesis and the potential clinical implications of its phase variable regulation have been outlined and discussed by O'Gara and Humphreys from Dublin. Further research in this area may contribute to the development of novel strategies for therapeutic intervention.

Finally, in addition to antibiotic prophylaxis, preventive strategies to control $S$ epidermidis medical-device-related infections are focusing on the development of improved biomaterials and physical electrical barriers to impede bacterial colonization.

FROM: O'Gara JP, Humphreys H. Staphylococcus epidermidis biofilms: importance and implications. $J$ Med Microbiol 2001;50:582-587. 